\title{
On the uniform convergence of double sine series
}

\author{
by \\ PÉTer Kórus and Ferenc Móricz (Szeged)
}

\begin{abstract}
Let a single sine series $(*) \sum_{k=1}^{\infty} a_{k} \sin k x$ be given with nonnegative coefficients $\left\{a_{k}\right\}$. If $\left\{a_{k}\right\}$ is a "mean value bounded variation sequence" (briefly, MVBVS), then a necessary and sufficient condition for the uniform convergence of series $(*)$ is that $k a_{k} \rightarrow 0$ as $k \rightarrow \infty$. The class MVBVS includes all sequences monotonically decreasing to zero. These results are due to S. P. Zhou, P. Zhou and D. S. Yu. In this paper we extend them from single to double sine series $(* *) \sum_{k=1}^{\infty} \sum_{l=1}^{\infty} c_{k l} \sin k x \sin l y$, even with complex coefficients $\left\{c_{k l}\right\}$. We also give a uniform boundedness test for the rectangular partial sums of series $(* *)$, and slightly improve the results on single sine series.
\end{abstract}

1. Introduction. Let $\left\{a_{k}: k=1,2, \ldots\right\}$ be a sequence of nonnegative real numbers (in symbols: $\left\{a_{k}\right\} \subset \mathbb{R}_{+}$), and consider the sine series

$$
\sum_{k=1}^{\infty} a_{k} \sin k x
$$

It is well-known (see, for example, [12, Vol. I, p. 182] that if the sequence $\left\{a_{k}\right\}$ is monotonically decreasing to zero, then series (1.1) converges for all $x$. On the other hand, according to the Cantor-Lebesgue theorem (see, for example, [1, p. 326]), if (1.1) converges on a set of positive measure, then $a_{k} \rightarrow 0$ as $k \rightarrow \infty$.

Chaundy and Joliffe [2] (see also [12, Vol. I, p. 182]) proved the following:

Theorem A. If $\left\{a_{k}\right\} \subset \mathbb{R}_{+}$is decreasing, then series (1.1) converges uniformly in $x$ if and only if

$$
k a_{k} \rightarrow 0 \text { as } k \rightarrow \infty .
$$

The monotonicity condition in Theorem A was relaxed by a number of authors (see, for example, the references in [4] and [11]). The following notion was introduced in [11], and it seems to be the most general one at present.

2000 Mathematics Subject Classification: Primary 42A20, 42A32, $42 B 99$.

Key words and phrases: double sine series, convergence in Pringsheim's sense, regular convergence, uniform convergence, uniform boundedness, mean value bounded variation double sequence, non-onesided bounded variation double sequence. 
A sequence $\left\{a_{k}\right\} \subset \mathbb{R}_{+}$is said to belong to the class MVBVS (= mean value bounded variation sequences) if there exist constants $C$ and $\lambda \geq 2$, both depending only on the sequence $\left\{a_{k}\right\}$, such that

$$
\sum_{k=m}^{2 m}\left|\Delta a_{k}\right| \leq \frac{C}{m} \sum_{k=\left[\lambda^{-1} m\right]}^{[\lambda m]} a_{k}, \quad m \geq \lambda,
$$

where $[\cdot]$ means the floor integral part of a real number and

$$
\Delta a_{k}:=a_{k}-a_{k+1}, \quad k=1,2, \ldots
$$

The following theorem was proved in [11, Theorem 5].

Theorem B. If $\left\{a_{k}\right\} \subset \mathbb{R}_{+}$belongs to the class MVBVS, then series (1.1) converges uniformly in $x$ if and only if condition (1.2) is satisfied.

We note that in [11] Theorem $B$ was proved in the following more general form: If $\left\{a_{k}\right\} \subset \mathbb{R}_{+}$belongs to the class MVBVS, then condition (1.2) is necessary and sufficient for the uniform convergence of series (1.1), and also for the continuity of its sum function. The addendum on the continuity of the sum function is a variant of a theorem of Paley [8] with almost the same proof. We recall that Paley's theorem says that if the Fourier coefficients of a continuous function $f$ are nonnegative, then the Fourier series of $f$ converges uniformly.

A particular case of the notion of MVBVS was earlier introduced in [9] as follows. A sequence $\left\{a_{k}\right\} \subset \mathbb{R}_{+}$is said to belong to the class NBVS (= non-onesided bounded variation sequences) if there exists a constant $C$, depending only on $\left\{a_{k}\right\}$, such that

$$
\sum_{k=m}^{2 m}\left|\Delta a_{k}\right| \leq C\left(a_{m}+a_{2 m}\right), \quad m=1,2, \ldots
$$

The following theorem was also formulated in [11] and supported with a counterexample.

Theorem C. If $\left\{a_{k}\right\} \subset \mathbb{R}_{+}$belongs to the class NBVS, then it belongs to MVBVS. The converse is not true.

Clearly, if $\left\{a_{k}\right\} \subset \mathbb{R}_{+}$is a decreasing sequence, then condition (1.4) is satisfied. Thus, Theorem A is an immediate corollary of Theorems B and C.

An analysis of the proofs in [9] and [11] reveals that the sufficiency part in Theorem $\mathrm{B}$ as well as Theorem $\mathrm{C}$ remain valid if we consider sequences $\left\{c_{k}: k=1,2, \ldots\right\}$ of complex numbers (in symbols: $\left\{c_{k}\right\} \subset \mathbb{C}$ ) and the sine series

$$
\sum_{k=1}^{\infty} c_{k} \sin k x,
$$

under appropriately modified conditions. 
Theorem $\mathrm{B}^{\prime}$. If $\left\{c_{k}\right\} \subset \mathbb{C}$ is such that

$$
k c_{k} \rightarrow 0 \quad \text { as } k \rightarrow \infty,
$$

and there exist constants $C$ and $\lambda \geq 2$ for which

$$
\sum_{k=m}^{2 m-1}\left|\Delta c_{k}\right| \leq \frac{C}{m} \sum_{k=\left[\lambda^{-1} m\right]}^{[\lambda m]}\left|c_{k}\right|, \quad m \geq \lambda,
$$

then series $\left(1.1^{\prime}\right)$ converges uniformly in $x$.

Theorem $\mathrm{C}^{\prime}$. If $\left\{c_{k}\right\} \subset \mathbb{C}$ is such that there exists a constant $C_{1}$ for which

$$
\sum_{k=m}^{2 m-1}\left|\Delta c_{k}\right| \leq C_{1}\left(\left|c_{m}\right|+\left|c_{2 m}\right|\right), \quad m=1,2, \ldots,
$$

then condition $\left(1.3^{\prime}\right)$ is satisfied for some constants $C$ and $\lambda \geq 2$.

We call the reader's attention to the upper limit of summation which is $2 m-1$ instead of $2 m$ on the left-hand sides in $\left(1.3^{\prime}\right)$ and $\left(1.4^{\prime}\right)$ (cf. (1.3) and (1.4)). However, this slight modification does not affect the proofs at all.

Finally, we note that analogous theorems can be proved for the uniform boundedness of the partial sums of sine series. For example, the scheme of the proofs of Theorems B and $\mathrm{B}^{\prime}$ can be modified so as to provide the following:

Theorem D. If $\left\{c_{k}\right\} \subset \mathbb{C}$ is such that condition $\left(1.3^{\prime}\right)$ is satisfied and

$$
\sup _{k \geq 1} k\left|c_{k}\right|<\infty,
$$

then

$$
\sup _{m \geq 1} \sup _{x}\left|\sum_{k=1}^{m} c_{k} \sin k x\right|<\infty .
$$

Conversely, if $\left\{c_{k}\right\} \subset \mathbb{R}_{+}$is such that conditions (1.3') and (1.6) are satisfied, then condition (1.5) is also satisfied.

2. Main results. We recall that the double series

$$
\sum_{k=1}^{\infty} \sum_{l=1}^{\infty} \gamma_{k l}
$$

of complex numbers is said to converge in Pringsheim's sense (see, for example, [12, Vol. II, pp. 301-302] if the finite limit of its rectangular partial sums

$$
\sum_{k=1}^{m} \sum_{l=1}^{n} \gamma_{k l}, \quad m, n=1,2, \ldots,
$$


exists as $m$ and $n$ tend to $\infty$ independently of each other. If, in addition, each "row series"

$$
\sum_{k=1}^{\infty} \gamma_{k l}, \quad l=1,2, \ldots,
$$

and each "column series"

$$
\sum_{l=1}^{\infty} \gamma_{k l}, \quad k=1,2, \ldots,
$$

converges, then (2.1) is said to converge regularly (see [3] and also [5]).

The Cauchy convergence criterion for regular convergence reads as follows (see [5]). The double series (2.1) converges regularly if and only if for every $\varepsilon>0$ there exists a natural number $m_{0}=m_{0}(\varepsilon)$ such that

$$
\left|\sum_{k=m}^{M} \sum_{l=n}^{N} \gamma_{k l}\right|<\varepsilon \quad \text { if } \max \{m, n\}>m_{0}, 1 \leq m \leq M, 1 \leq n \leq N .
$$

It is easy to see that it is enough to require (2.2) to hold in the special cases when either $m>m_{0}$ and $n=1$, or $n>m_{0}$ and $m=1$.

After these preliminaries, let $\left\{c_{k l}: k, l=1,2, \ldots\right\}$ be a double sequence of complex numbers (in symbols: $\left\{c_{k l}\right\} \subset \mathbb{C}$ ), and consider the double sine series

$$
\sum_{k=1}^{\infty} \sum_{l=1}^{\infty} c_{k l} \sin k x \sin l y
$$

We use the standard notations for the difference operators:

$$
\begin{aligned}
\Delta_{10} c_{k l} & :=c_{k l}-c_{k+1, l}, \quad \Delta_{01} c_{k l}:=c_{k l}-c_{k, l+1}, \\
\Delta_{11} c_{k l} & :=\Delta_{01}\left(\Delta_{10} c_{k l}\right)=\Delta_{10}\left(\Delta_{01} c_{k l}\right) \\
& =c_{k l}-c_{k+1, l}-c_{k, l+1}+c_{k+1, l+1}, \quad k, l=1,2, \ldots
\end{aligned}
$$

We recall that a double sequence $\left\{c_{k l}\right\}$ is said to be monotonically decreasing if

$$
c_{k l} \geq 0, \quad \Delta_{10} c_{k l} \geq 0, \quad \Delta_{01} c_{k l} \geq 0, \quad \Delta_{11} c_{k l} \geq 0
$$

for all $k, l=1,2, \ldots$ It is clear that if

$$
c_{k l} \rightarrow 0 \quad \text { as } k+l \rightarrow \infty,
$$

then we have

$$
\begin{gathered}
\Delta_{10} c_{m n}=\sum_{l=n}^{\infty} \Delta_{11} c_{m l}, \quad \Delta_{01} c_{m n}=\sum_{k=m}^{\infty} \Delta_{11} c_{k n}, \\
c_{m n}=\sum_{k=m}^{\infty} \sum_{l=n}^{\infty} \Delta_{11} c_{k l}, \quad m, n=1,2, \ldots
\end{gathered}
$$


Consequently, under condition (2.5), the first three inequalities in (2.4) follow from the fourth one.

The second author proved in [6] that if $\left\{c_{j k}\right\} \subset \mathbb{C}$ is such that condition (2.5) is satisfied and $\left\{c_{j k}\right\}$ is of bounded variation in the sense that

$$
\sum_{k=1}^{\infty} \sum_{l=1}^{\infty}\left|\Delta_{11} c_{k l}\right|<\infty,
$$

then the double series (2.3) converges regularly for all $(x, y)$. In particular, if the double sequence $\left\{c_{k l}\right\}$ of nonnegative numbers (in symbols: $\left\{c_{k l}\right\} \subset$ $\left.\mathbb{R}_{+}\right)$is monotonically decreasing to zero, then the double sine series (2.3) converges regularly for all $(x, y)$.

On the other hand, according to the two-dimensional extension of the Cantor-Lebesgue theorem (see [7]), if series (2.3) converges regularly on a set of positive (planar) measure, then we necessarily have (2.5).

The two-dimensional extension of the classical theorem of Chaundy and Joliffe (see Theorem A in Section 1), proved by Zhak and Shnel̆der [10], reads as follows.

TheOREM E. If $\left\{c_{k l}\right\} \subset \mathbb{R}_{+}$is a monotonically decreasing double sequence, then the regular convergence of the double sine series (2.3) is uniform in $(x, y)$ if and only if

$$
k l c_{k l} \rightarrow 0 \quad \text { as } k+l \rightarrow \infty .
$$

Our goal is to relax the monotonicity condition in Theorem E. To this end, we introduce the class MVBVDS motivated by $\left(1.3^{\prime}\right)$. A double sequence $\left\{c_{k l}\right\} \subset \mathbb{C}$ is said to belong to the class MVBVDS (= mean value bounded variation double sequences) if there exist constants $C$ and $\lambda \geq 2$, both depending only on $\left\{c_{k l}\right\}$, such that

$$
\begin{gathered}
\sum_{k=m}^{2 m-1}\left|\Delta_{10} c_{k n}\right| \leq \frac{C}{m} \sum_{k=\left[\lambda^{-1} m\right]}^{[\lambda m]}\left|c_{k n}\right|, \quad m \geq \lambda, n=1,2, \ldots, \\
\sum_{l=n}^{2 n-1}\left|\Delta_{01} c_{m l}\right| \leq \frac{C}{n} \sum_{l=\left[\lambda^{-1} n\right]}^{[\lambda n]}\left|c_{m l}\right|, \quad n \geq \lambda, m=1,2, \ldots, \\
\sum_{k=m}^{2 m-1} \sum_{l=n}^{2 n-1}\left|\Delta_{11} c_{k l}\right| \leq \frac{C}{m n} \sum_{k=\left[\lambda^{-1} m\right]}^{[\lambda m]} \sum_{l=\left[\lambda^{-1} n\right]}^{[\lambda n]}\left|c_{k l}\right|, \quad m, n \geq \lambda .
\end{gathered}
$$

Our first main result is a two-dimensional extension of Theorems B and $\mathrm{B}^{\prime}$. 
Theorem 1. If $\left\{c_{k l}\right\} \subset \mathbb{C}$ belongs to the class MVBVDS and condition (2.7) is satisfied, then the regular convergence of the double sine series (2.3) is uniform in $(x, y)$.

Conversely, if $\left\{c_{k l}\right\} \subset \mathbb{R}_{+}$belongs to the class MVBVDS and the regular convergence of (2.3) is uniform in $(x, y)$, then condition (2.7) is satisfied.

In particular, the following corollary of Theorem 1 follows immediately: If $\left\{c_{k l}\right\} \subset \mathbb{R}_{+}$belongs to the class MVBVDS, then the regular convergence of the double sine series (2.3) is uniform in $(x, y)$ if and only if condition (2.7) is satisfied.

To our best knowledge, an extension of Paley's theorem mentioned in Section 1 (see [8]) from single to double Fourier series with nonnegative Fourier coefficients is not known. Thus, regarding the extension of the addendum to Theorem B in Section 1, on continuity of the sum function of (2.3), we raise the following problem:

Problem. Is the following claim true or false? If $\left\{c_{k l}\right\} \subset \mathbb{R}_{+}$belongs to the class MVBVDS, then condition (2.7) is necessary or sufficient for the continuity of the sum function of the double sine series (2.3).

The introduction of the following class of double sequences of complex numbers is motivated by $\left(1.4^{\prime}\right):\left\{c_{k l}\right\} \subset \mathbb{C}$ is said to belong to the class NBVDS (non-onesided bounded variation double sequences) if there exists a constant $C$, depending only on $\left\{c_{k l}\right\}$, such that

$$
\begin{aligned}
& \sum_{k=m}^{2 m-1}\left|\Delta_{10} c_{k n}\right| \leq C\left(\left|c_{m n}\right|+\left|c_{2 m, n}\right|\right), \\
& \sum_{l=n}^{2 n-1}\left|\Delta_{01} c_{m l}\right| \leq C\left(\left|c_{m n}\right|+\left|c_{m, 2 n}\right|\right), \\
& \sum_{k=m}^{2 m-1} \sum_{l=n}^{2 n-1}\left|\Delta_{11} c_{k l}\right| \leq C\left(\left|c_{m n}\right|+\left|c_{2 m, n}\right|+\left|c_{m, 2 n}\right|+\left|c_{2 m, 2 n}\right|\right), \\
& m, n=1,2, \ldots
\end{aligned}
$$

Our second main result is a two-dimensional extension of Theorems C and $\mathrm{C}^{\prime}$.

ThEOREM 2. If $\left\{c_{k l}\right\} \subset \mathbb{C}$ belongs to the class NBVDS, then it belongs to MVBVDS. The converse is not true.

Clearly, if $\left\{c_{k l}\right\} \subset \mathbb{R}_{+}$is a monotonically decreasing double sequence, then conditions (2.11)-(2.13) are satisfied. Thus, Theorem E, which was proved by Zhak and Shnelder [10] in another way, is an immediate corollary of Theorems 1 and 2 . 
So far, we have presented sufficient conditions for the regular convergence of the double sine series $(2.3)$ to be uniform in $(x, y)$. It is of some interest to observe that the conditions in Theorem 1 can be easily reformulated so as to guarantee the uniform boundedness of the rectangular sums

$$
\begin{aligned}
& s(m, M ; n, N ; x, y):=\sum_{k=m}^{M} \sum_{l=n}^{N} c_{k l} \sin k x \sin l y, \\
& 1 \leq m \leq M, 1 \leq n \leq N .
\end{aligned}
$$

The reformulated Theorem 1 reads as follows.

Theorem 3. If $\left\{c_{k l}\right\} \subset \mathbb{C}$ belongs to the class MVBVDS and

$$
\sup _{k, l \geq 1} k l\left|c_{k l}\right|<\infty
$$

then

$$
\sup _{1 \leq m \leq M, 1 \leq n \leq N} \sup _{x, y}|s(m, M ; n, N ; x, y)|<\infty .
$$

Conversely, if $\left\{c_{k l}\right\} \subset \mathbb{R}_{+}$belongs to the class MVBVDS and condition (2.16) is satisfied, then (2.15) also holds.

The following corollary of Theorem 3 is immediate: If $\left\{c_{k l}\right\} \subset \mathbb{R}_{+}$belongs to the class MVBVDS, in particular, if $\left\{c_{k l}\right\}$ is a monotonically decreasing double sequence, then a necessary and sufficient condition for the uniform boundedness of the rectangular sums (2.14) is that the double sequence $\left\{k l_{k l}\right\}$ is bounded.

We note that each of the theorems and corollaries in this section can be easily extended to multiple sine series with complex or nonnegative real coefficients, respectively. However, the technical details become more cumbersome.

3. Auxiliary results. To prove Theorems $1-3$, we will need four lemmas, which may also be useful in other contexts. In addition, we present a proof of Theorem $\mathrm{C}^{\prime}$.

Lemma 1. If $\left\{c_{k l}\right\} \subset \mathbb{C}$ is such that conditions (2.7) and (2.10) are satisfied, then

$$
m n \sum_{k=m}^{\infty} \sum_{l=n}^{\infty}\left|\Delta_{11} c_{k l}\right| \rightarrow 0 \quad \text { as } m+n \rightarrow \infty \text { and } m, n \geq 2 \lambda .
$$

Proof. Given $\varepsilon>0$, by (2.7) there exists a natural number $m_{1}=m_{1}(\varepsilon)$ such that

$$
k l\left|c_{k l}\right|<\varepsilon \quad \text { for all } m+n>m_{1}, m, n \geq 2 \lambda .
$$

Without loss of generality, we may assume that $m_{1} \geq 4 \lambda$. 
Making use of (2.10) and (3.2) gives

$$
\begin{aligned}
\sum_{k=m}^{\infty} \sum_{l=n}^{\infty}\left|\Delta_{11} c_{k l}\right| & =\sum_{i=0}^{\infty} \sum_{j=0}^{\infty} \sum_{2^{i}} \sum_{m \leq k<2^{i+1}} \sum_{2^{j} n \leq l<2^{j+1} n}\left|\Delta_{11} c_{k l}\right| \\
& \leq C \sum_{i=0}^{\infty} \sum_{j=0}^{\infty} \frac{1}{2^{i+j} m n} \sum_{k=\left[\lambda^{-1} 2^{i} m\right]}^{\left[\lambda 2^{i} m\right]} \sum_{l=\left[\lambda^{-1} 2^{j} n\right]}^{\left[\lambda 2^{j} n\right]}\left|c_{k l}\right| \\
& \leq \frac{C \varepsilon}{m n} \sum_{i=0}^{\infty} \sum_{j=0}^{\infty} \frac{1}{2^{i+j}} \sum_{k=\left[\lambda^{-1} 2^{i} m\right]}^{\left[\lambda 2^{i} m\right]} \sum_{l=\left[\lambda^{-1} 2^{j} n\right]}^{\left[\lambda 2^{j} n\right]} \frac{1}{k l} .
\end{aligned}
$$

We have

$$
\begin{aligned}
\sum_{k=\left[\lambda^{-1} 2^{i} m\right]}^{\left[\lambda 2^{i} m\right]} \frac{1}{k} & \leq \frac{1}{\left[\lambda^{-1} 2^{i} m\right]}+\int_{\left[\lambda^{-1} 2^{i} m\right]}^{\left[\lambda 2^{i} m\right]} \frac{d x}{x} \\
& \leq 1+\ln \frac{\left[\lambda 2^{i} m\right]}{\left[\lambda^{-1} 2^{i} m\right]} \leq 1+\ln \left(2 \lambda^{2}\right), \quad i=0,1,2, \ldots,
\end{aligned}
$$

since

$$
\frac{\left[\lambda 2^{i} m\right]}{\left[\lambda^{-1} 2^{i} m\right]} \leq \frac{\lambda 2^{i} m}{\lambda^{-1} 2^{i} m-1} \leq 2 \lambda^{2} \quad \text { for } m \geq 2 \lambda .
$$

Now $(3.3),(3.4)$ and its counterpart with $1 / l$ in place of $1 / k$ yield

$$
m n \sum_{k=m}^{\infty} \sum_{l=n}^{\infty}\left|\Delta_{11} c_{k l}\right| \leq C \varepsilon\left(1+\ln \left(2 \lambda^{2}\right)\right)^{2}
$$

whenever $m+n \geq m_{1}$ and $m, n \geq 2 \lambda$. Since $\varepsilon>0$ is arbitrary, this proves (3.1).

LEMma 2. Under the conditions of Lemma 1, we have

$$
m n \sum_{k=m}^{\infty} \sup _{l \geq n}\left|\Delta_{10} c_{k l}\right| \rightarrow 0
$$

and

$$
m n \sum_{l=n}^{\infty} \sup _{k \geq m}\left|\Delta_{01} c_{k l}\right| \rightarrow 0 \quad \text { as } m+n \rightarrow \infty, m, n \geq 2 \lambda .
$$

Proof. By (2.6),

$$
\sup _{l \geq n}\left|\Delta_{10} c_{k l}\right|=\sup _{l \geq n}\left|\sum_{l_{1}=l}^{\infty} \Delta_{11} c_{k l_{1}}\right| \leq \sum_{l=n}^{\infty}\left|\Delta_{11} c_{k l}\right|, \quad k, n=1,2, \ldots
$$

Thus, we have

$$
\sum_{k=m}^{\infty} \sup _{l \geq n}\left|\Delta_{10} c_{k l}\right| \leq \sum_{k=m}^{\infty} \sum_{l=n}^{\infty}\left|\Delta_{11} c_{k l}\right|
$$

and an application of Lemma 1 yields (3.5). A similar argument gives (3.6). 
Lemma 3. If $\left\{c_{k l}\right\} \subset \mathbb{R}_{+}$belongs to the class MVBVDS, then

$$
m n c_{m n} \leq(8 C+2) \sum_{k=[m / 2 \lambda]}^{[\lambda m]} \sum_{t=[n / 2 \lambda]}^{[\lambda n]} c_{k l}, \quad m, n \geq 2 \lambda,
$$

where the constants $C$ and $\lambda$ are from (2.7)-(2.9).

Proof. Let $m \geq 2 \lambda$ and $\nu$ be arbitrary natural numbers. Applying inequality (2.8) for $\mu=m+1, m+2, \ldots, 2 m$ gives

$$
\begin{aligned}
c_{m \nu} & =\sum_{k=m}^{\mu-1} \Delta_{10} c_{k \nu}+c_{\mu \nu} \leq \sum_{k=m}^{\mu-1}\left|\Delta_{10} c_{k \nu}\right|+c_{\mu \nu} \\
& \leq \sum_{k=[\mu / 2]}^{2[\mu / 2]-1}\left|\Delta_{10} c_{k \nu}\right|+c_{\mu \nu} \leq \frac{C}{[\mu / 2]} \sum_{k=\left[\lambda^{-1}[\mu / 2]\right]}^{[\lambda[\mu / 2]]} c_{k \nu}+c_{\mu \nu} \\
& \leq \frac{2 C}{m} \sum_{k=[m / 2 \lambda]}^{[\lambda m]} c_{k \nu}+c_{\mu \nu}, \quad m<\mu \leq 2 m, \nu \geq 1 .
\end{aligned}
$$

Next, let $n \geq 2 \lambda$ and $\mu$ be arbitrary natural numbers. An analogous argument, using inequality $(2.9)$ for $\nu=n+1, n+2, \ldots, 2 n$, gives

$$
c_{\mu n} \leq \frac{2 C}{n} \sum_{l=[n / 2 \lambda]}^{[\lambda n]} c_{\mu l}+c_{\mu \nu}, \quad \mu \geq 1, n<\nu \leq 2 n .
$$

Finally, let $m, n \geq 2 \lambda$ be arbitrary natural numbers. A double version of the above argument, using (2.10) for $\mu=m+1, m+2, \ldots, 2 m$ and $\nu=n+1, n+2, \ldots, 2 n$, gives

$$
\begin{aligned}
c_{m n} & =\sum_{k=m}^{\mu-1} \sum_{l=n}^{\nu-1} \Delta_{11} c_{k l}+c_{m \nu}+c_{\mu n}-c_{\mu \nu} \\
& \leq \sum_{k=m}^{\mu-1} \sum_{l=n}^{\nu-1}\left|\Delta_{11} c_{k l}\right|+c_{m \nu}+c_{\mu n} \\
& \leq \sum_{k=[\mu / 2]}^{2[\mu / 2]-1} \sum_{l=[\nu / 2]}^{2[\nu / 2]-1}\left|\Delta_{11} c_{k l}\right|+c_{m \nu}+c_{\mu n} \\
& \leq \frac{C}{[\mu / 2][\nu / 2]} \sum_{k=\left[\lambda^{-1}[\mu / 2]\right]}^{[\lambda[\mu / 2]]} \sum_{l=\left[\lambda^{-1}[\nu / 2]\right]}^{[\lambda[\nu / 2]]} c_{k l}+c_{m \nu}+c_{\mu n} \\
& \leq \frac{4 C}{m n} \sum_{k=[m / 2 \lambda]}^{[\lambda m]} \sum_{l=[n / 2 \lambda]}^{[\lambda n]} c_{k l}+c_{m \nu}+c_{\mu n}, \\
&
\end{aligned}
$$


If we add up all the inequalities in (3.10), then we obtain

$$
m n c_{m n} \leq 4 C \sum_{k=[m / 2 \lambda]}^{[\lambda m]} \sum_{l=[n / 2 \lambda]}^{[\lambda n]} c_{k l}+m \sum_{\nu=n+1}^{2 n} c_{m \nu}+n \sum_{\mu=m+1}^{2 m} c_{\mu n} .
$$

Now making use of (3.8) and (3.9) yields

$$
\begin{aligned}
m n c_{m n} \leq & 4 C \sum_{k=[m / 2 \lambda]}^{[\lambda m]} \sum_{l=[n / 2 \lambda]}^{[\lambda n]} c_{k l}+2 C \sum_{k=[m / 2 \lambda]}^{[\lambda m]} \sum_{\nu=n+1}^{2 n} c_{k \nu} \\
& +2 C \sum_{\mu=m+1}^{2 m} \sum_{l=[n / 2 \lambda]}^{[\lambda n]} c_{\mu l}+2 \sum_{\mu=m+1}^{2 m} \sum_{\nu=n+1}^{2 n} c_{\mu \nu} \\
\leq & (8 C+2) \sum_{k=[m / 2 \lambda]}^{[\lambda m]} \sum_{l=[n / 2 \lambda]}^{[\lambda n]} c_{k l},
\end{aligned}
$$

which is (3.7).

In Section 1, we have noted that the first statement in Theorem $\mathrm{C}$ has not been explicity proved in [11]. Instead, it is only indicated there that the proof is similar to that of [11, Proposition 3]. For the reader's convenience, below we present a proof of the more general Theorem $\mathrm{C}^{\prime}$. This proof moreover illustrates the key ingredients of the proof of our Theorem 2 in Section 4.

Proof of Theorem $C^{\prime}$. Let $m \geq 6$. We apply inequality $\left(1.4^{\prime}\right)$ for all integers between $m-[m / 6]$ and $m+[m / 6]$. Adding up these inequalities yields

$$
\sum_{i=m-[m / 6]}^{m+[m / 6]} \sum_{k=i}^{2 i-1}\left|\Delta c_{k}\right| \leq C \sum_{i=m-[m / 6]}^{m+[m / 6]}\left(\left|c_{i}\right|+\left|c_{2 i}\right|\right) .
$$

It is easy to see that the term $\left|\Delta c_{k}\right|$ with $m \leq k \leq 2 m-1$ occurs on the left-hand side of (3.11) at least

$$
\left[\frac{m}{6}\right]+1 \geq \frac{m}{6} \quad \text { times. }
$$

Furthermore, on the right-hand side of (3.11), the smallest index of the term $\left|c_{i}\right|$ is

$$
m-\left[\frac{m}{6}\right] \geq \frac{m}{3},
$$

while the largest index of the term $\left|c_{2 i}\right|$ does not exceed

$$
2\left(m+\left[\frac{m}{6}\right]\right) \leq 3 m ;
$$


and over the range $m-[m / 6] \leq i \leq m+[m / 6]$, we have

$$
\max i \leq m+\left[\frac{m}{6}\right]<2\left(m-\left[\frac{m}{6}\right]\right) \leq \min 2 i .
$$

Keeping these observations in mind, from (3.11) it follows that

$$
\frac{m}{6} \sum_{k=m}^{2 m-1}\left|\Delta c_{k}\right| \leq C \sum_{i=[m / 3]}^{3 m}\left|c_{i}\right| \text {. }
$$

Thus, we have shown that inequality $\left(1.3^{\prime}\right)$ is satisfied with $6 C$ in place of $C$ and $\lambda=3$. Consequently, $\left\{c_{k}\right\}$ belongs to the class MVBVS.

For the second statement in Theorem $\mathrm{C}$, the counterexample given in [11, Proposition 4] is recalled in the following

Lemma 4. Let $\left\{b_{k}\right\} \subset \mathbb{R}_{+}$be an arbitrary decreasing sequence. Define

$$
c_{k}:= \begin{cases}0 & \text { for } 2^{i} \leq k<2^{i}+i \\ b_{k} & \text { for } 2^{i}+i \leq k<2^{i+1}-i, \\ 0 & \text { for } 2^{i+1}-i \leq k<2^{i+1}, i=1,2, \ldots\end{cases}
$$

Then the sequence $\left\{c_{k}\right\}$ belongs to the class MVBVS, but not to NBVS.

\section{Proofs of Theorems $1-3$}

Proof of Theorem 1. Part 1. By conditions (2.8) and (2.9), Theorem $\mathrm{C}^{\prime}$ applies to the single sequences

$$
\sum_{k=1}^{\infty} c_{k l} \sin k x, \quad l=1,2, \ldots, \quad \sum_{l=1}^{\infty} c_{k l} \sin l y, \quad k=1,2, \ldots,
$$

and guarantees that each row series and each column series of the double series (2.3) converge uniformly in $(x, y)$.

In order to prove the uniform regular convergence of (2.3), we consider the rectangular sums

$$
s(m, M ; n, N ; x, y):=\sum_{k=m}^{M} \sum_{l=n}^{N} c_{k l} \sin k x \sin l y, \quad 1 \leq m<M, 1 \leq n<N .
$$

It is enough to consider the case where $0<x, y<\pi$. Let

$$
\mu(x):=\left[\frac{1}{x}\right] \quad \text { and } \quad \nu(y):=\left[\frac{1}{y}\right] .
$$

We distinguish the following four cases. 
CASe (a): $1 \leq m<M \leq \mu(x)$ and $1 \leq n<N \leq \nu(y)$. A trivial estimate gives

$$
\begin{aligned}
|s(m, M ; n, N ; x, y)| & \leq x y \sum_{k=m}^{M} \sum_{l=n}^{N} k l\left|c_{k l}\right| \\
& \leq \frac{1}{\mu(x) \nu(y)} \sum_{k=1}^{\mu(x)} \sum_{l=1}^{\nu(y)} k l\left|c_{k l}\right| .
\end{aligned}
$$

By (2.7), the double sequence $\left\{k l c_{k l}\right\}$ boundedly converges to zero. Therefore, the arithmetic mean on the right-hand side of (4.2) converges to zero as $\mu(x), \nu(y) \rightarrow \infty$.

CASE (b): $\mu(x)<m<M$ and $1 \leq n<N \leq \nu(y)$. This time, we estimate as follows:

$$
\begin{aligned}
|s(m, M ; n, N ; x, y)| & =\sum_{l=n}^{N}|\sin l y| \cdot\left|\sum_{k=m}^{M} c_{k l} \sin k x\right| \\
& \leq \sum_{l=n}^{N} l y\left|\sum_{k=m}^{M} c_{k l} \sin k x\right| \\
& \leq \frac{1}{\nu(y)} \sum_{l=n}^{\nu(y)} l\left|\sum_{k=m}^{M} c_{k l} \sin k x\right| .
\end{aligned}
$$

Summation by parts gives

$$
\sum_{k=m}^{M} c_{k l} \sin k x=\sum_{k=m}^{M-1} \widetilde{D}_{k}(x) \Delta_{10} c_{k l}+c_{M l} \widetilde{D}_{M}(x)-c_{m l} \widetilde{D}_{m-1}(x),
$$

where $\widetilde{D}_{k}(x)$ is the conjugate Dirichlet kernel (see, for example, [12, Vol. I, p. 49]):

$$
\widetilde{D}_{k}(x):=\sum_{i=1}^{k} \sin i x=\frac{\cos \frac{x}{2}-\cos \left(k+\frac{1}{2}\right) x}{2 \sin \frac{x}{2}} .
$$

Using the familiar estimate

$$
\left|\widetilde{D}_{k}(x)\right| \leq \pi / x, \quad k=1,2, \ldots, 0<x<\pi,
$$

it follows from (4.3) and (4.4) that

$$
\begin{aligned}
|s(m, M ; n, N ; x, y)| & \leq \frac{\pi}{x \nu(y)} \sum_{l=n}^{\nu(y)} l\left\{\sum_{k=m}^{M-1}\left|\Delta_{10} c_{k l}\right|+\left|c_{M l}\right|+\left|c_{m l}\right|\right\} \\
& \leq \frac{\pi m}{\nu(y)} \sum_{l=n}^{\nu(y)} l\left\{\sum_{k=m}^{\infty}\left|\Delta_{10} c_{k l}\right|+2 \sup _{k \geq m}\left|c_{k l}\right|\right\} .
\end{aligned}
$$


By Lemma 2 (cf. (3.5)), the arithmetic mean on the right-hand side of (4.5) converges to zero as $m, n \rightarrow \infty$.

CASE (c): $1 \leq m<M \leq \mu(x)$ and $\nu(y)<n<N$. This is the symmetric counterpart of Case (b), and it can be handled analogously. The corresponding estimate is

$$
|s(m, M ; n, N ; x, y)| \leq \frac{\pi n}{\mu(x)} \sum_{k=1}^{\mu(x)} k\left\{\sum_{l=n}^{\infty}\left|\Delta_{01} c_{k l}\right|+2 \sup _{l \geq n}\left|c_{k l}\right|\right\} .
$$

By Lemma 2 (cf. (3.6)), the right-hand side of (4.6) converges to zero as $m, n \rightarrow \infty$.

CASE (d): $\mu(x)<m<M$ and $\nu(y)<n<N$. This time, we perform double summation by parts to obtain

$$
\begin{aligned}
s(m, M ; n, N ; x, y) & =\sum_{k=m}^{M-1} \sum_{l=n}^{N-1} \widetilde{D}_{k}(x) \widetilde{D}_{l}(y) \Delta_{11} c_{k l} \\
& +\sum_{k=m}^{M-1} \widetilde{D}_{k}(x) \widetilde{D}_{N}(y) \Delta_{10} c_{k N}-\sum_{k=m}^{M-1} \widetilde{D}_{k}(x) \widetilde{D}_{n-1}(y) \Delta_{10} c_{k n} \\
& +\sum_{l=n}^{N-1} \widetilde{D}_{M}(x) \widetilde{D}_{l}(y) \Delta_{01} c_{M l}-\sum_{l=n}^{N-1} \widetilde{D}_{m-1}(x) \widetilde{D}_{l}(y) \Delta_{01} c_{m l} \\
& +c_{M N} \widetilde{D}_{M}(x) \widetilde{D}_{N}(y)-c_{m N} \widetilde{D}_{m-1}(x) \widetilde{D}_{N}(y) \\
& -c_{M n} \widetilde{D}_{M}(x) \widetilde{D}_{n-1}(y)+c_{m n} \widetilde{D}_{m-1}(x) \widetilde{D}_{n-1}(y)
\end{aligned}
$$

whence it follows that

$$
\begin{aligned}
|s(m, M ; n, N ; x, y)| \leq \frac{\pi^{2}}{x y}\left\{\sum_{k=m}^{M-1} \sum_{l=n}^{N-1}\left|\Delta_{11} c_{k l}\right|\right. \\
\left.\quad+\sum_{k=m}^{M-1}\left(\left|\Delta_{10} c_{k N}\right|+\left|\Delta_{10} c_{k n}\right|\right)+\sum_{l=n}^{N-1}\left|\Delta_{01} c_{M l}\right|+\left|\Delta_{01} c_{m l}\right|\right) \\
\left.\quad+\left|c_{M N}\right|+\left|c_{m N}\right|+\left|c_{M n}\right|+\left|c_{m n}\right|\right\} \\
\leq \pi^{2} m n\left\{\sum_{k=m}^{\infty} \sum_{l=n}^{\infty}\left|\Delta_{11} c_{k l}\right|+2 \sum_{k=m}^{\infty} \sup _{l \geq n}\left|\Delta_{10} c_{k l}\right|\right. \\
\left.\quad+2 \sum_{l=n}^{\infty} \sup _{k \geq m}\left|\Delta_{01} c_{k l}\right|+4 \sup _{k \geq m, l \geq n}\left|c_{k l}\right|\right\} .
\end{aligned}
$$

By Lemmas 1 and 2, the right-hand side of (4.7) converges to zero as $m, n$ $\rightarrow \infty$. 
After these preliminaries, we can prove the uniform regular convergence of the double sine series (2.3) as follows. By (2.8) and (2.9), Theorem $\mathrm{C}^{\prime}$ implies the uniform convergence of each row series and each column series of (2.3).

By (2.7), for any $\varepsilon>0$ there exists a natural number $m_{1}=m_{1}(\varepsilon) \geq 2 \lambda$ such that

$$
k l\left|c_{k l}\right|<\varepsilon \quad \text { for all } k, l>m_{1} ;
$$

consequently, there exists $m_{2}=m_{2}(\varepsilon) \geq 2 \lambda$ such that

$$
\left|\frac{1}{m n} \sum_{k=1}^{m} \sum_{l=1}^{n} k l c_{k l}\right|<\varepsilon \quad \text { for all } m, n>m_{2} .
$$

By Lemma 1, there exists $m_{3}=m_{3}(\varepsilon) \geq 2 \lambda$ such that

$$
m n \sum_{k=m}^{\infty} \sum_{l=n}^{\infty}\left|\Delta_{11} c_{k l}\right|<\varepsilon \quad \text { for all } m, n>m_{3} .
$$

By Lemma 2, we also have

$$
m n \sum_{k=m}^{\infty} \sup _{l \geq n}\left|\Delta_{10} c_{k l}\right|<\varepsilon
$$

and

$$
m n \sum_{l=n}^{\infty} \sup _{k \geq m}\left|\Delta_{01} c_{k l}\right|<\varepsilon \quad \text { for all } m, n \geq m_{3} ;
$$

consequently, there exists $m_{4}=m_{4}(\varepsilon) \geq 2 \lambda$ such that

$$
\frac{m}{N} \sum_{l=n}^{N} l \sum_{k=m}^{\infty}\left|\Delta_{10} c_{k l}\right|<\varepsilon \quad \text { for all } m, n>m_{4} \text { and } N>n ;
$$

and

$$
\frac{n}{M} \sum_{k=m}^{M} k \sum_{l=n}^{\infty}\left|\Delta_{01} c_{k l}\right|<\varepsilon \quad \text { for all } m, n>m_{4} \text { and } M>m .
$$

Let $m_{0}:=\max \left\{m_{1}, m_{2}, m_{3}, m_{4}\right\}$. We claim that

$$
\begin{aligned}
|s(m, M ; n, N ; x, y)|<\left(9 \pi^{2}+6 \pi+1\right) \varepsilon \quad \text { for all }(x, y) \\
\text { whenever } M>m>m_{0} \text { and } N>n>m_{0} .
\end{aligned}
$$

To justify this claim, given $0<x, y<\pi$, we distinguish nine cases listed under (i)-(ix). We recall that $\mu(x)$ and $\nu(y)$ were defined in (4.1).

(i) $m_{0}<m<M \leq \mu(x)$ and $m_{0}<n<N \leq \nu(y)$. This was treated in Case (a). By (4.2), (4.8) and (4.9), we have (4.15) with $\varepsilon$ in place of $\left(9 \pi^{2}+6 \pi+1\right) \varepsilon$. 
(ii) $\mu(x)<m<M$ and $m_{0}<n<N \leq \nu(y)$. This was treated in Case (b). By (4.5), (4.9) and (4.13), we have (4.15) with $3 \pi \varepsilon$.

(iii) $m_{0}<m \leq \mu(x)<M$ and $m_{0}<n<N \leq \nu(y)$. This can be split into two subcases which belong to Case (a) and Case (b), respectively. Combining it with the previous cases (i) and (ii) gives (4.15) with $(3 \pi+1) \varepsilon$.

(iv) $m_{0}<m<M \leq \mu(x)$ and $\nu(y)<n<N$. This was indicated in Case (c), and it is the symmetric counterpart of (ii). Thus, we have (4.15) again with $3 \pi \varepsilon$.

(v) $\mu(x)<m<M$ and $\nu(y)<n<N$. This was treated in Case (d). By (4.7), (4.8), (4.10)-(4.12), we deduce (4.15) with $\pi^{2}(1+2+2+4) \varepsilon=9 \pi^{2} \varepsilon$.

(vi) $m_{0}<m \leq \mu(x)<M$ and $\nu(y)<n<N$. This can be split into two cases which belong to Case (c) and Case (d), respectively. Combining it with case (v) and case (ix) below gives $(4.15)$ with $\left(9 \pi^{2}+3 \pi+1\right) \varepsilon$.

(vii) $m_{0}<m<M \leq \mu(x)$ and $m_{0}<n \leq \nu(y)<N$. This can be split into two cases which belong to Case (a) and Case (c), respectively; and it is the symmetric counterpart of (iii). Thus, we have (4.15) again with $(3 \pi+1) \varepsilon$.

(viii) $\mu(x)<m<M$ and $m_{0}<n \leq \nu(y)<N$. This can be split into four subcases which fall under Cases (a)-(d), respectively. Combining it with the previous cases (i), (ii), (iv) and (v) gives (4.15) with the upper bound as indicated.

(ix) $m_{0}<m \leq \mu(x)<M$ and $m_{0}<n \leq \nu(y)<N$. This is Case (c), and it is the symmetric counterpart of (iii). Thus, we have (4.15) again with $(3 \pi+1) \varepsilon$.

Now the justification of (4.15) is complete. What we have proved so far is that the inequality for $|s(m, M ; n, N ; x, y)|$ in (4.15) holds when $\min \{m, n\}$ $>m_{0}$. In order to complete the proof of the uniform regular convergence of the double sine series (2.3), we need to prove an analogous inequality when just $\max \{m, n\}$ is large enough.

To this end, fix $\eta>0$. We choose $\varepsilon>0$ in (4.15) to be

$$
\varepsilon:=\frac{\eta}{2\left(9 \pi^{2}+6 \pi+1\right)},
$$

and focus on the first $m_{0}$ row series and the first $m_{0}$ column series in (2.3). Each of them converges uniformly in $(x, y)$. Therefore, there exists a natural number $n_{1}=n_{1}(\eta)$ such that

$$
\begin{array}{r}
|s(m, M ; n, N ; x, y)|<\eta / 2 \quad \text { for all }(x, y) \text { whenever } \\
\text { either } n_{1}<m \leq M \text { and } 1 \leq n \leq N \leq m_{0}, \\
\quad \text { or } 1 \leq m \leq M \leq m_{0} \text { and } n_{1}<n \leq N .
\end{array}
$$

Let $n_{0}:=\max \left\{m_{0}, n_{1}\right\}$. Putting together (4.15) and (4.16) yields $|s(m, M ; n, N ; x, y)|<\eta \quad$ for all $(x, y)$ whenever either $n_{0}<m \leq M$ and $1 \leq n \leq N$, or $1 \leq m \leq M$ and $n_{0}<n \leq N$. 
Taking into account the remark made after (2.2) in Section 2 and that $\eta>0$ is arbitrary, this proves the uniform regular convergence of the double sine series (2.3).

This completes the proof of Part 1 .

Part 2. By (2.8), (2.9) and the uniform convergence of each row series and each column series (2.3), we may apply the necessity part of Theorem B to deduce (2.7) when either $k \rightarrow \infty$ and $l$ is fixed, or vice versa.

It remains to prove (2.7) when both $k$ and $l$ tend to $\infty$. To this end, we will apply Lemma 3 . Let $m, n \geq 4 \lambda$ and set

$$
x(m):=\frac{\pi}{2 \lambda m} \quad \text { and } \quad y(n):=\frac{\pi}{2 \lambda n} .
$$

Clearly, for all $k$ in the range $[m / 2 \lambda] \leq k \leq[\lambda m]$ we have

$$
k x(m) \leq \lambda m \frac{\pi}{2 \lambda m}=\frac{\pi}{2}
$$

and

$$
k x(m) \geq\left(\frac{m}{2 \lambda}-1\right) \frac{\pi}{2 \lambda m} \geq \frac{m}{4 \lambda} \frac{\pi}{2 \lambda m}=\frac{\pi}{8 \lambda^{2}},
$$

since $m \geq 4 \lambda$. Likewise,

$$
\frac{\pi}{8 \lambda^{2}} \leq l y(n) \leq \frac{\pi}{2} \quad \text { whenever } \quad\left[\frac{n}{2 \lambda}\right] \leq l \leq[\lambda n] .
$$

By these inequalities and the assumption that all $c_{k l}$ are nonnegative, it follows from (3.7) that

$$
\begin{aligned}
\sum_{k=[m / 2 \lambda]}^{[\lambda m]} \sum_{l=[n / 2 \lambda]}^{[\lambda n]} c_{k l} \sin k x(m) & \sin l y(n) \\
& \geq\left(\sin \frac{\pi}{8 \lambda^{2}}\right)^{2} \sum_{k=[m / 2 \lambda]}^{[\lambda m]} \sum_{l=[n / 2 \lambda]}^{[\lambda n]} c_{k l} \\
& \geq \frac{1}{8 C+2}\left(\sin \frac{\pi}{8 \lambda^{2}}\right)^{2} m n c_{m n} .
\end{aligned}
$$

Since the regular convergence of the double sine series (2.3) is supposed to be uniform in $(x, y)$, the fulfillment of $(2.7)$ when $k, l \rightarrow \infty$ is an immediate consequence of (4.17).

This completes the proof of Part 2.

Now the proof of Theorem 1 is complete.

Proof of Theorem 2. Part 1. By Theorem $\mathrm{C}^{\prime}$, conditions (2.11) and (2.12) imply (2.8) and (2.9), respectively. Thus, in order to prove that the double sequence $\left\{c_{k l}\right\}$ in question belongs to the class MVBVDS, it remains to check (2.10). 
To this end, let $m, n \geq 6$ be given. We apply inequality (2.13) for all integers $i$ and $j$ in the ranges

$$
m-\left[\frac{m}{6}\right] \leq i \leq m+\left[\frac{m}{6}\right] \quad \text { and } n-\left[\frac{n}{6}\right] \leq j \leq n+\left[\frac{n}{6}\right] .
$$

Adding up all these inequalities results in

$$
\begin{aligned}
& \sum_{i=m-[m / 6]}^{m+[m / 6]} \sum_{j=n-[n / 6]}^{n+[n / 6]} \sum_{k=i}^{2 i-1} \sum_{l=j}^{2 j-1}\left|\Delta_{11} c_{k l}\right| \\
& \leq C \sum_{i=m-[m / 6]}^{m+[m / 6]} \sum_{j=n-[n / 6]}^{n+[n / 6]}\left(\left|c_{i j}\right|+\left|c_{2 i, j}\right|+\left|c_{i, 2 j}\right|+\left|c_{2 i, 2 j}\right|\right) .
\end{aligned}
$$

We observe that for the integers $k$ and $l$ in the ranges

$$
m \leq k \leq 2 m-1 \text { and } n \leq l \leq 2 n-1,
$$

the term $\left|\Delta c_{k l}\right|$ occurs on the left-hand side of (4.19) at least

$$
\left(\left[\frac{m}{6}\right]+1\right)\left(\left[\frac{n}{6}\right]+1\right) \geq \frac{m n}{36} \quad \text { times. }
$$

Furthermore, on the right-hand side of (4.19), the smallest indices of the term $\left|c_{i j}\right|$ are

$$
m-\left[\frac{m}{6}\right] \geq \frac{m}{3} \quad \text { and } \quad n-\left[\frac{n}{6}\right] \geq \frac{n}{3} ;
$$

while the largest indices of the term $\left|c_{2 i, 2 j}\right|$ do not exceed

$$
2\left(m+\left[\frac{m}{6}\right]\right) \leq 3 m \text { and } 2\left(n+\left[\frac{n}{6}\right]\right) \leq 3 n ;
$$

and over the ranges indicated in (4.18), we have

$$
\max i \leq m+\left[\frac{m}{6}\right]<2\left(m-\left[\frac{m}{6}\right]\right) \leq \min 2 i,
$$

and analogous inequalities for $j$.

Taking these observations into account, from (4.19) it follows that

$$
\frac{m n}{36} \sum_{k=m}^{2 m-1} \sum_{l=n}^{2 n-1}\left|\Delta_{11} c_{k l}\right| \leq C \sum_{k=[m / 3]}^{3 m} \sum_{l=[n / 3]}^{3 n}\left|c_{k l}\right| .
$$

Thus, we have shown that the required inequality (2.13) is satisfied with $36 \mathrm{C}$ in place of $C$ and $\lambda=3$. Consequently, $\left\{c_{k l}\right\}$ belongs to the class MVBVDS.

Part 2. Take the sequence $\left\{c_{k}\right\}$ given in Lemma 4 and define the double sequence $\left\{c_{k l}\right\}$ by setting $c_{k l}:=c_{k} c_{l}, k, l=1,2, \ldots$ Since $\left\{c_{k}\right\}$ belongs to 
the class MVBVS, we have

$$
\begin{aligned}
\sum_{k=m}^{2 m-1}\left|\Delta_{10} c_{k n}\right| & =c_{n} \sum_{k=m}^{2 m-1}\left|\Delta c_{k}\right| \\
& \leq c_{n} \frac{C}{m} \sum_{k=\left[\lambda^{-1} m\right]}^{[\lambda m]} c_{k}=\frac{C}{m} \sum_{k=\left[\lambda^{-1} m\right]}^{[\lambda m]} c_{k n},
\end{aligned}
$$

that is, condition (2.8) is satisfied. Condition (2.9) can be proved analogously.

It is also clear that

$$
\begin{aligned}
& \sum_{k=m}^{2 m-1} \sum_{l=n}^{2 n-1}\left|\Delta_{11} c_{k l}\right|=\sum_{k=m}^{2 m-1}\left|\Delta c_{k}\right| \sum_{l=n}^{2 n-1}\left|\Delta c_{l}\right| \\
& \leq \frac{C}{m} \sum_{k=\left[\lambda^{-1} m\right]}^{[\lambda m]} c_{k} \cdot \frac{C}{n} \sum_{l=\left[\lambda^{-1} n\right]}^{[\lambda n]} c_{l}=\frac{C^{2}}{m n} \sum_{k=\left[\lambda^{-1} m\right]}^{[\lambda m]} \sum_{l=\left[\lambda^{-1} n\right]}^{[\lambda n]} c_{k l},
\end{aligned}
$$

which is (2.10) with $C^{2}$ in place of $C$. To sum up, we have proved that $\left\{c_{k l}\right\}$ belongs to the class MVBVDS.

On the other hand, $\left\{c_{k l}\right\}$ cannot belong to NBVDS. It is easy to see that none of conditions (2.11)-(2.13) is satisfied. Again, the justification hinges on the fact that we can factorize both sides of the inequalities in question exactly in the same way as in the cases of (4.21) and (4.22).

The proof of Theorem 2 is complete.

Proof of Theorem 3. It goes along the same lines as that of Theorem 1 with appropriate modifications.

Acknowledgements. The authors express their gratitude to the referee for valuable suggestions to improve the presentation of the results.

This research was supported by the Hungarian National Foundation for Scientific Research under Grant T 046192.

\section{References}

[1] G. Alexits, Convergence Problems of Orthogonal Series, Pergamon Press, Oxford, 1961.

[2] T. W. Chaundy and A. E. Joliffe, The uniform convergence of a certain class of trigonometrical series, Proc. London Math. Soc. 15 (1916), 214-216.

[3] G. H. Hardy, On the convergence of certain multiple series, Proc. Cambridge Philos. Soc. 19 (1916-1919), 86-95.

[4] L. Leindler, Necessary and sufficient conditions for uniform convergence and boundedness of a general class of sine series, Austral. J. Math. Anal. Appl. 4 (2007), no. 1, art. 10, 4 pp. 
[5] F. Móricz, Some remarks on the notion of regular convergence of multiple series, Acta Math. Acad. Sci. Hungar. 41 (1983), 161-168.

[6] —, Convergence and integrability of double trigonometric series with coefficients of bounded variation, Proc. Amer. Math. Soc. 102 (1988), 633-640.

[7] —, The Cantor-Lebesgue and Denjoy-Luzin properties for double systems of functions, Anal. Math. 17 (1991), 297-305.

[8] R. E. A. C. Paley, On Fourier series with positive coefficients, J. London Math. Soc. 7 (1932), 205-208.

[9] D. S. Yu and S. P. Zhou, A generalization of the monotonicity condition and applications, Acta Math. Hungar. 115 (2007), 247-267.

[10] I. E. Zhak and A. A. Shneĭder, Conditions for uniform convergence of double sine series, Izv. Vyssh. Uchebn. Zaved. Mat. 1966, no. 4 (53), 44-52 (in Russian).

[11] S. P. Zhou, P. Zhou and D. S. Yu, Ultimate generalization to monotonicity for uniform convergence of trigonometric series, http://arxiv.org/abs/math/0611805v1.

[12] A. Zygmund, Trigonometric Series, Cambridge Univ. Press, 1959.

University of Szeged

Bolyai Institute

Aradi vértanúk tere 1

6720 Szeged, Hungary

E-mail: Korus.Peter@stud.u-szeged.hu

moricz@math.u-szeged.hu

Received September 23, 2008

Revised version December 9, 2008 